\title{
Ovarian Cancer and Primary Peritoneal Carcinoma pN1 TNM Finding v7
}

National Cancer Institute

\section{Source}

National Cancer Institute. Ovarian Cancer and Primary Peritoneal Carcinoma pN1 TNM

Finding v7. NCI Thesaurus. Code C89657.

Ovarian cancer or primary peritoneal carcinoma with regional lymph node metastasis.

(from AJCC 7th Ed.) 